\title{
Robust Image Watermarking Algorithm Based on Radon and Analytic Fourier-Mellin Transforms
}

\author{
Jie Zhao*
}

School of Electronic Information and Electrical Engineering, Shangluo University, Shangluo, Shaanxi, 726000, China

\begin{abstract}
The most existing digital watermarking methods were robust to general attacks such as additive noise, image filtering, JPEG compression and so on, but resisting to geometric attacks is still a difficult challenge. This paper proposed a watermarking algorithm resisting to geometrical attacks. The Radon transform was employed to project two dimension image onto projection space. Then the rotation of this image was converted to a translation of the projection. At the same time, the scaling was converted to a scaling of the projection besides the scaling of the projection amplitude. After that, the analytic Fourier-Mellin transform was used to construct invariant moments. These invariant moments are used to design and detect watermark. The experiment demonstrates the proposed method was robust to usually image processing operation, and performed robust to geometrical attacks.
\end{abstract}

Keywords: Image watermarking, Radon transform, Analytic Fourier-Mellin transform, Invariant moments.

\section{INTRODUCTION}

Internet and multimedia techniques provide great convenience for obtain the information. Meanwhile, the multimedia security problem is outstanding. The problem is growing more and more prominent because of the development of cloud computing and Internet of things. In order to solve the copyright protection and content authentication problem, the digital watermark technology was proposed and got rapid development in recent years.

Digital image watermark technology is commonly achieved by certain modifications to the host signal. According to the embedding domain, spatial and transform domain are two types of the most traditional image watermark technology [1]. Spatial watermarking method embeds watermark information into original digital image, while transform domain method performs watermark embedding in transform domain. Moreover, the methods based on ICA, vector quantization were proposed $[2,3]$.

Recently, watermarking methods robust to geometric attacks is becoming a hot issue. The exhaustive search algorithm was used early. The main drawback of this method is the computation complexity, and it is sensitive to attacks [4]. Tao $\mathrm{H}$ et al. proposed the template insertion algorithm to resisting to geometrical attacks [5]. Its disadvantage is that the applied templates are eliminated easily by the attack and the applied various geometric transformations are unable to be recognized during the template detection process. The log-polar mapping is used in [6]. It is useful for scaling and rotation distortion, but it is not effective to cropping operation. The main drawback is the quality damage of image by

\footnotetext{
*Address correspondence to this author at the School of Electronic Information and Electrical Engineering, Shangluo University, Shangluo, Shaanxi, 726000, China; Tel: 13992467763; E-mail: slxyzj@163.com
}

the log-polar mapping. Some proposed several methods using geometrically invariant image features. Lecture [7] used circular Hough transform to achieve the watermark synchronization. The circular Hough transform was adopted to construct the invariant features to geometrical attacks.

A few of image watermarking algorithms using image histogram were proposed [8]. Pun et al. obtained geometric invariant watermarking method based on histogram in wavelet domain [9]. H.Y. Lee et al. proposed a new algorithm by scale-invariant feature transform (SIFT). It generated several circular patches through image feature points and the watermark was embedded [10]. This method is useful to geometrical distortions. The low similarity is the main drawback. In [11], authors presented a novel algorithm based on SVD. The authors proposed an effective method based on Zemike moments [12]. But they only got the results of rotation invariant. In [13], the Tchebycheff moments are used to get the rotation and scaling invariant. Image reconstruction is required for these methods. The complexity and large calculating quantity are notable. C. Deng et al. proposed a novel method based on elliptical regions formed by Harris-Affine detector [14]. The regions are normalized into circular ones. The watermark information was embedded into these normalized regions. It is robust to geometric attacks. However, it performs badly for common signal processing operations. A. Masoud and H. Ahmed proposed a novel algorithm based on $\mathrm{Hu}$ moment and affine moment [15]. It is simple and effective against geometric attacks. On the other hand, the fidelity of the image is worse and this method is sensitive to cropping operation, since it requires biggish amplitude factor in order to distinguish the original carrier image and the watermarked one in detection process. Given above reasons, this paper proposes a novel image watermarking method resisting to scaling and rotation attacks. It conducts imperceptible revision to the carrier image and does not use moments for image reconstruction after embedding the water- 


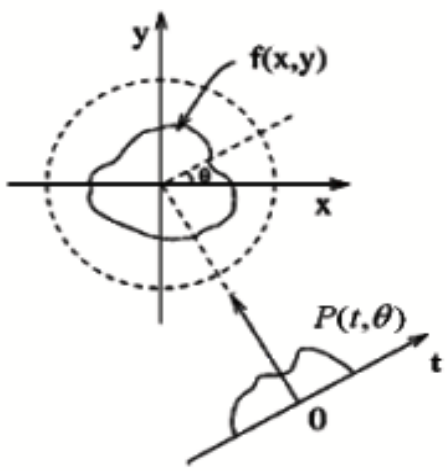

Fig. (1). The Radon transform of a two dimension function $f(x, y)$.

mark. A few of invariant moments are used to determine whether the watermark exists when detecting the watermark.

\section{INVARIANT ANALYSIS}

\subsection{Radon Transform}

The Radon transform of a two dimension image $f(x, y)$ is given as:

$R(t, \theta)\{f(x, y)\}=P(t, \theta)$

$=\iint f(x, y) \delta(t-x \cos \theta-y \sin \theta) d x d y$

where $\delta(t)$ is the Dirac function, $t$ is the perpendicular distance of a straight line from the origin $O$ (see Fig. 1), $\theta$ is the angle between the distance vector and the x-axis, i.e., $\theta \in[0, \pi)[16]$.

The Radon transform has useful properties about image translation, scaling and rotation.

About image translation:

The angle dependent translates along the spatial variable of the projection when the translation of $f(x, y)$ occurred:

$R(t, \theta)\left\{f\left(x-x_{0}, y-y_{0}\right)\right\}=P\left(t-t_{0}, \theta\right)$

$P(t, \theta)$ is the Radon transform of $f(x, y)$, and $t_{0}=x_{0} \cos \theta+y_{0} \sin \theta$.

About image scaling:

The scaling of $f(x, y)$ in the image space leads to a scaling of the projections in the spatial variable besides the scaling of the projection amplitude:

$$
R(t, \theta)\left\{f\left(\frac{x}{\lambda}, \frac{y}{\lambda}\right)\right\}=\lambda P\left(\frac{t}{\lambda}, \theta\right), \lambda \neq 0
$$

About image rotation:

A rotation of $f(x, y)$ with angle $\varphi \in[0,2 \pi)$ results in a circular shift of the projection along the variable $\theta$ :

$$
R(t, \theta)\left\{f_{\varphi}(x, y)\right\}=P(t, \theta+\varphi)
$$

$f_{\varphi}(x, y)$ is the rotated image of original image $f(x, y)$ with angle $\varphi$.

\subsection{Analytic Fourier-Mellin Transform}

The analytic Fourier-Mellin transform is given by:

$M(s, k)=\int_{0}^{\infty} \int_{0}^{2 \pi} g(r, \theta) \cdot r^{s-1} \cdot e^{-i k \theta} d r d \theta$

where $g(r, \theta)$ is the polar coordinates representation of a two dimension image. After the Fourier-Mellin transform, $g(r, \theta)$ became $M(s, k) . s=\sigma-i u, u \in R, \sigma$ is positive real constant and its value was recommended to be around 0.5 [17]. The analytic Fourier-Mellin transform of $g(r, \theta)$ is redefined by:

$M(u, k)=\int_{0}^{\infty} \int_{0}^{2 \pi} g(r, \theta) \cdot r^{\sigma-i u-1} \cdot e^{-i k \theta} d r d \theta$

\subsection{The Invariant Function}

Let $P(r, \theta)$ dedicate the Radon transform of image $f(x, y)$, and $f_{g}(x, y)$ be the zoomed and rotated image of $f(x, y)$. The scale factor is $\lambda$ and the rotation angle is $\varphi$. After that, the Radon transform of $f_{g}(x, y)$ can be written as:

$P_{g}(r, \theta)=\lambda P(r / \lambda, \theta+\varphi)$

On the basis of above, the analytic Fourier-Mellin transform of $P_{g}(r, \theta)$ can be written as: 


$$
\begin{aligned}
& M_{g}(u, k)=\int_{0}^{\infty} \int_{0}^{2 \pi} P_{g}(r, \theta) \cdot r^{\sigma-i u-1} \cdot e^{-i k \varphi} d r d \theta \\
& =\int_{0}^{\infty} \int_{0}^{2 \pi} \lambda P(r / \lambda, \theta+\varphi) r^{\sigma-i u-1} e^{-i k \varphi} d r d \theta \\
& \quad \text { Let } \tau=r / \lambda, \beta=\theta+\varphi, \text { then } \\
& M_{g}(u, k)=\int_{0}^{\infty} \int_{0}^{2 \pi} \lambda^{2} P(\tau, \beta)(\lambda \tau)^{\sigma-i u-1} e^{-i k(\beta-\varphi)} d \tau d \beta \\
& =\lambda^{\sigma-i u+1} e^{i k \varphi} \int_{0}^{\infty} \int_{0}^{2 \pi} P(\tau, \beta) \tau^{\sigma-i u-1} e^{-i k \beta} d \tau d \beta \\
& =\lambda^{\sigma+1} \lambda^{-i u} e^{i k \varphi} \int_{0}^{\infty} \int_{0}^{2 \pi} P(\tau, \beta) \tau^{\sigma-i u-1} e^{-i k \beta} d \tau d \beta \\
& =\lambda^{\sigma-i u+1} e^{i k \varphi} M(u, k)
\end{aligned}
$$

where, $M(u, k)$ is the analytic Fourier-Mellin transform of $P(r, \theta)$. And then $Z(u, k)$ is defined to achieve the scaling and rotation invariance [18]. It is given by:

$$
Z(u, k)=M(0,0)^{-(\sigma-i u+1) /(\sigma+1)} e^{i k a r g(M(0,1))} M(u, k)
$$

where $M(0,0)$ is the Fourier-Mellin transform of $P(r, \theta)$ with $u$ and $k$ being zero. Such as $M(0,1)$. According to $P_{g}(r, \theta), Z_{g}(u, k)$ can be written by:

$$
\begin{aligned}
& Z_{g}(u, k)=M_{g}(0,0)^{-(\sigma-i u+1) /(\sigma+1)} e^{i k \arg \left(M_{g}(0,1)\right)} M_{g}(u, k) \\
& =\left[\lambda^{\sigma+1} M(0,0)\right]^{\frac{-(\sigma+i u)}{\sigma+1}} e^{i k a r g\left[\lambda^{\sigma+1} e^{i k \varphi} M(0,1)\right]} \lambda^{\sigma-i u+1} e^{i k \varphi} M(u, k) \\
& =M(0,0)^{-(\sigma-i u+1) /(\sigma+1)} e^{i k \arg (M(0,1))} M(u, k)=Z(u, k)
\end{aligned}
$$

The above equation shows that $Z(u, k)$ is scaling and rotation invariant. On the basis of above result, the invariant features could be extracted from $Z(u, k)$. The paper [10] proposed energy, deviation, and entropy as invariant features. In order to obtain general means, the low order geometric moments of $Z(u, k)$ were used in this paper.

\section{WATERMARK DESIGN}

A new function $f$ is defined, and $f$ should meet the tolerance condition of $d-\varepsilon \leq f \leq d+\varepsilon$. $d$ is a numerical value predefined in advance, which is related to the carrier image. $\varepsilon$ indicates the system tolerance. And then the original carrier image should be changed to reach the condition. For example, the original image $I$ can be modified by $\bar{I}=I+\alpha \log (I)$ where $\alpha$ is weighting coefficient. In this experimental process, $f$ is designed as the mean of the $\log$ value of low order geometric moments about $Z(u, k)$. When detecting the watermark, the mean of low order geometric moments of $Z(u, k)$ of test image were calculated. It declared that the watermark is existed if $|f-d| \leq \varepsilon$. Otherwise, it means the unauthentic.

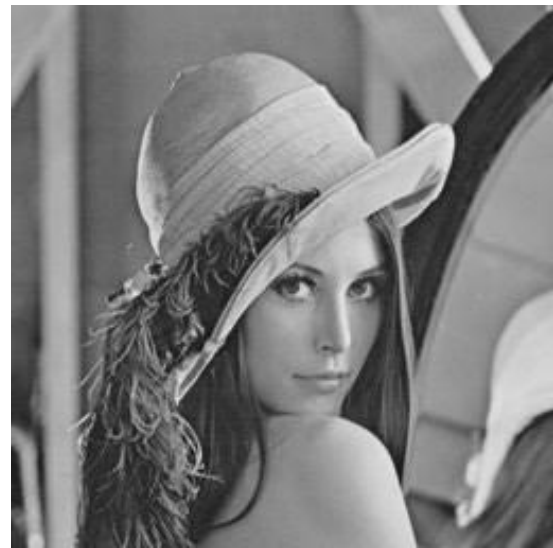

Fig. (2). Original image.

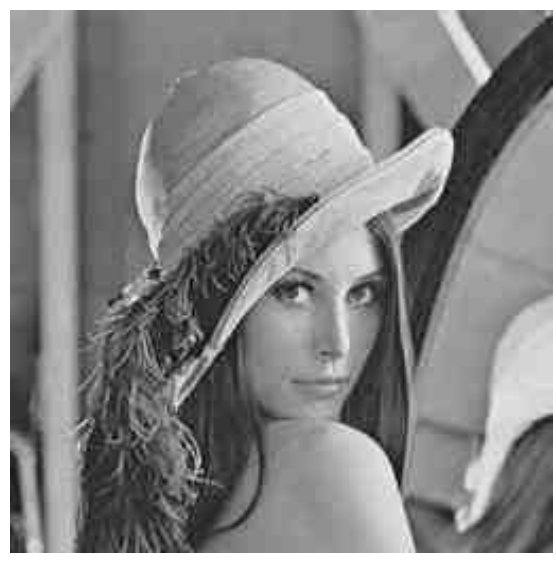

Fig. (3). Watermarked image.

Gray image of "Lena" is used as the original image. The original image and modified image are shown in Fig. (2) and Fig. (3). $\alpha=1.9$ and $d=12.1$, then the watermark was constructed while the mean value of moments of watermarked image was 12.1478. The exhaustive search was adopted with a short step size. There is contradiction between the robustness and the invisibility of watermark system. Then a tradeoff was required and it was explained before. The special nonlinear weighted function of original image was defined. Then the watermarked image is modified by adding the weighted value. The contrast is the difference between them and this difference is small. The difference is invisible to human eyes since $\alpha$ is a small factor.

Table 1 shows the test results of this method under common attacks. The results show that this method is effective. In addition, the proposed watermarking scheme works as an authentication system. It presents the yes/no answers of authenticity. Hence, it can be considered as a 1-bit watermarking system. The detection process only needs to know the nominal values and which could be agreed upon before.

\section{CONCLUSION}

A robust image watermarking algorithm used Radon transform and analytic Fourier-Mellin transform is proposed in this paper. The simulation results show that the method is robust to image filtering, additive noise, JPEG compression, 
Table 1. Results of detecting.

\begin{tabular}{|c|c|c|c|}
\hline Attack & $f$ & $|f-d|$ & Detection \\
\hline Watermarked original & 12.1478 & 0.0478 & pass \\
\hline Gaussian noise & 12.1482 & 0.0482 & pass \\
\hline Salt \& Pepper noise & 12.1481 & 0.0481 & pass \\
\hline Rotation $20^{\circ}$ & 12.1030 & 0.0030 & pass \\
\hline Rotation $-20^{\circ}$ & 12.1038 & 0.0038 & pass \\
\hline Scaling 50\% & 11.9638 & 0.1362 & pass \\
\hline Scaling $200 \%$ & 12.2423 & 0.1423 & pass \\
\hline Median filtering & 12.1480 & 0.0480 & pass \\
\hline Mean filtering & 12.1465 & 0.0465 & pass \\
\hline $\operatorname{JPEG}(\mathrm{Q}=50)$ & 12.1485 & 0.0485 & pass \\
\hline $\mathrm{JPEG}(\mathrm{Q}=25)$ & 12.1483 & 0.0483 & pass \\
\hline Cropping 10\% & 12.0121 & 0.0879 & pass \\
\hline Cropping 25\% & 11.8441 & 0.2559 & fail \\
\hline
\end{tabular}

geometrical attacks as rotation and scaling. The proposed image watermarking system is implemented without image reconstruction, and this leads to the simplicity. It is a practical and reliable method. Meanwhile, it does not require original carrier image in detecting process.

\section{CONFLICT OF INTEREST}

The author confirms that this article content has no conflict of interest.

\section{ACKNOWLEDGEMENTS}

This work is supported by the Science and Technology Foundation Project of Shangluo University (NO. 13SKY015).

\section{REFERENCES}

R. Ahuja, S. S. Bedi, and H. Agarwal, "A survey of digital watermarking scheme", MIT International Journal of Computer Science and Information Technology, vol. 2, no. 1, pp. 52-59, 2012.
[2] T. V. Nguyen, and J. C. Patra, "A simple ICA-based digital image watermarking scheme", Digital Signal Processing, vol. 18, no. 5, pp. $762-776,2008$

[3] J.J. Shena, and J.M. Renb, "A robust associative watermarking technique based on vector quantization”, Digital Signal Processing, vol. 20 , no. 5, pp. 1408-1423, 2010.

[4] P. Singh, and R. S. Chadha, "A survey of digital watermarking techniques, applications and attacks", International Journal of Engineering and Innovative Technology, vol. 2, no. 9, pp. 165-175, 2013

[5] H. Tao, L. Chongmin, J. M. Zain, and A. N. Abdalla, "Robust image watermarking theories and techniques: a review", Journal of Applied Research and Technology, vol. 12, no. 1, pp. 122-138, 2014.

[6] D. Zheng, J. Zhao, and A. E. Saddik, "RST-invariant digital image watermarking based on log-polar mapping and phase correlation", IEEE Transactions on Circuits and Systems for Video Technology, vol. 13, no. 8, pp. 753-756, 2003.

[7] H.Y. Lee, C.H. Lee, and H.K. Lee, "Geometrically invariant watermarking: synchronization through circular Hough transform", Multimedia Tools and Applications, vol. 34, no. 3, pp. 337-353, 2007.

[8] H. A. Ali, and S.A.K. Khamis, "Robust digital image watermarking technique based on histogram analysis", World of Computer Science and Information Technology Journal, vol. 2, no. 5, pp. 163$168,2012$.

[9] C.M. Pun, and X.C. Yuan, "Geometric invariant digital image watermarking scheme based on histogram in DWT domain", Journal of Multimedia, vol. 5, no. 5, pp. 434-442, 2010.

[10] H.Y. Lee, H.S. Kim, and H.K. Lee, "Robust image watermarking using local invariant features", Optical Engineering, vol. 45, no. 3 , pp. 1931-1934, 2006.

[11] X. Jia, Y. Qi, L. Shao, and X. Jia, "A watermark algorithm based on SVD and image geometric correction", International Conference on Systems and Informatics, vol. 19-20, pp. 1861-1865, 2012.

[12] H.S. Kim, and H.K. Lee, "Invariant image watermark using Zernike moments", IEEE Transactions on Circuits and Systems for Video Technology, vol. 13, no. 8, pp. 766-775, 2003.

[13] C. Deng, X. Gao, X. Li, and D. Tao, "A local Tchebycheff moments-based robust image watermarking", Signal Processing, vol 89, no. 8, pp. 1531-1539, 2009.

[14] C. Deng, X. Gao, D. Tao, and X. Li, "Geometrically invariant watermarking using affine covariant regions", IEEE International Conference on Image Processing, vol. 12-15, pp. 413-416, 2008.

[15] A. Masoud, and H. Ahmed, "Geometric Invariance in Image Watermarking", IEEE Transactions on Image Processing, vol. 13, no. 2, pp. 145-153, 2004.

[16] P.R. Galigekere, M.N.S. Swamy, A. Fenster and D.W. Holdsworth, "Moment patterns in the radon space", Optical Engineering, vol. 39, no. 4, pp. 1088-1097, 2000.

[17] S. Derrode, and F. Ghorbel, "Robust and efficient Fourier-Mellin transform approximations for gray-level image reconstruction and complete invariant description", Computer Vision Image and Understanding, vol. 83, no. 1, pp. 57-78, 2001.

[18] X. Wang, B. Xiao, J. F. Ma, and X.L. Bi, "Scaling and rotation invariant analysis approach to object recognition based on Radon and Fourier-Mellin transforms", Pattern Recognition, vol. 40, no. 12, pp. 3503-3508, 2007.

(C) Jie Zhao; Licensee Bentham Open.

This is an open access article licensed under the terms of the Creative Commons Attribution Non-Commercial License (http://creativecommons.org/licenses/ by-nc/4.0/) which permits unrestricted, non-commercial use, distribution and reproduction in any medium, provided the work is properly cited. 